\title{
Sniffing out the evidence; It's now time for public health bodies to recognize the link between COVID-19 and smell and taste disturbance*
}

\author{
Jerome R. Lechien ${ }^{1,2}$, Claire Hopkins ${ }^{3, \#}$, Sven Saussez ${ }^{4, \#}$ \\ 'COVID-19 Task Force of the Young-Otolaryngologists of the International Federations of Oto-rhino-laryngological Societies \\ (YO-IFOS) \\ ${ }^{2}$ Department of Human Anatomy and Experimental Oncology, Faculty of Medicine, UMONS Research Institute for Health \\ Sciences and Technology, University of Mons (UMons), Mons, Belgium \\ ${ }^{3}$ Guy's and St Thomas NHS Foundation Trust, London, UK \\ ${ }^{4}$ Department of Otorhinolaryngology and Head and Neck Surgery, CHU de Bruxelles, CHU Saint-Pierre, School of Medicine, \\ Université Libre de Bruxelles, Brussels, Belgium
}

\section{Rhinology 58: 4, 402 - 403, 2020}

https://doi.org/10.4193/Rhin20.159

*Received for publication:

April 15, 2020

Accepted: April 27, 2020

" have contributed as co-senior authors
Since the outbreak of the pandemic, anecdotal observations have been accumulating rapidly that sudden anosmia and dysgeusia are peculiar symptoms associated with the COVID-19 infection. Prof C. Hopkins, as President of British Rhinological Society, published a letter describing "the loss of sense of smell as a marker of COVID-19 infection" and proposed that adults presenting with anosmia but no other symptoms should self-isolate for seven days. The Hopkins team published the first case report and case series as well as other evidence that isolated sudden onset anosmia (ISOA), should be considered highly suspicious for SARS-CoV-2 ${ }^{(1)}$. Subsequently, a larger series of 2428 patients presenting with new onset anosmia during the COVID-19 pandemic has been reported, of whom $16 \%$ report loss of sense of smell as an isolated symptom. Only $51 \%$ reported the recognized symptoms of cough or fever. A major limitation of this series however, was a lack of access to testing to confirm the COVID-19 status of the patients ${ }^{(2)}$; in the 80 who had been tested $74 \%$ were positive. In the same way, the American Academy of Otolaryngology-head and neck surgery (AA0-HNS) proposed "that anosmia could be added to the list of screening tools for possible COVID-19 infection. More, they warrant serious consideration for self-isolation and testing those patients".

The Young Physicians Group of the International Federation of Otorhinolaryngological Societies (YO-IFOS), published the first prospective study, using a questionnaire based on the smell and taste component of the National Health and Nutrition Examination Survey, and the short version of Questionnaire of Olfactory Disorders-Negative Statements (QOD-NS). This European multicentric study reported on 417 mild-to-moderate COVID-19 positive patients and highlights that $85.6 \%$ and $88.0 \%$ reported olfactory and gustatory dysfunctions, respectively. Interestingly, olfactory dysfunction appeared before (11.8\%), after (65.4\%) or at the same time (22.8\%) as the appearance of general or ENT symptoms ${ }^{(3)}$. Using the same questionnaire, we have initiated a larger survey for French, Belgian, Italian and Spanish populations; in this series of 1420 mild to moderate COVID+ patients, our Bayesian analysis identified that reported anosmia was one of the most prevalent symptoms, reported in $70.2 \%$ of our COVID-19+ patients (accepted for publication J Int Med). Kaye et al. report on 237 US patients with COVID-19 and anosmia, collated through an AAO-HNS resource, and state that loss of sense of smell was present before diagnosis in $73 \%$ of respondents, and was the initial symptom in $26.6 \%^{(4)}$, supporting the suggestion it is a useful early marker to prompt testing or self-isolation. Interestingly, these early reports have suggested a predominance of olfactory dysfunction in younger, female patients; this is in contrast to the general population, where olfactory function has been found to be lower in males an older patients ${ }^{(5,6)}$.

Two as yet unpublished studies under-going peer review have specifically reported on the rate of COVID19 infection in otherwise paucisymptomatic patients with anosmia as the major/ only symptom. Lechien et al. ${ }^{(7)}$ found that $87 \%$ of patients tested within 12 days of onset of anosmia had a positive viral load for COVID-19, with viral load decreasing throughout the period from onset of anosmia. Similarly, Salmon et al. have taken undertaken PCR analysis of nasopharyngeal swabs from 55 patients within 7 days of developing sudden onset anosmia without nasal obstruction; 94\% were COVID-19 positive ${ }^{(8)}$. 
Subsequent to initial reports suggesting anosmia could be a symptom of COVID-19, loss of sense of smell was added to a symptom tracker app developed by a team at King's College London, with 1.5 million app users between 24-29 March 2020. Of these, 1,702 reported having been tested for COVID-19, with 579 positive results and 1,123 negative results. 59\% of COVID-19 positive patients reported loss of smell and taste, compared with only $18 \%$ of those who tested negative ${ }^{(9)}$. These results were much stronger in predicting a positive COVID-19 diagnosis than self-reported fever. Using all the data collected, the King's research team developed a model to identify which combination of symptoms together could predict COVID-19 cases which includes a combination of loss of smell and taste, fever, persistent cough, fatigue, diarrhea, abdominal pain and loss of appetite. The strongest predictor is loss of smell and taste. In a similar study, from a single centre in the US ${ }^{(10)}$, reporting on 59 COVID-19 positive patients, and 203 with negative test results, smell and taste impairment were independently and strongly associated with COVID-19 positivity (OR 10.9 for anosmia and 10.2 for ageusia). Combining these 2 studies, anosmia has a positive predictive value of $61 \%$ for COVID19 positivity on PCR testing, sensitivity of $60 \%$ and specificity of $81 \%$.

While selection bias likely exists amongst volunteers for online surveys, studies of consecutive cases of COVID-19 positive patients provide similar estimates of prevalence; Spinato et al., interviewing mild-moderate patients ${ }^{(11)}$, found that $64 . \%$ reported reduction in smell or taste, $75 \%$ of whom reported a severe or complete loss. Most recently, the first report of objective testing of olfactory function in a case-matched controlled series of patients with COVID-19 in Iran has been reported ${ }^{(12)} ; 59$ out of 60 patients were found to have olfactory dysfunction, with significantly poorer olfactory function that controls $(P<0.0001)$. The majority of patients were completely anosmic.

There is a known high seasonal variation in presentation in olfactory dysfunction with a peak in $\mathrm{March}^{(13)}$, likely caused by viruses that cause the common cold; caution therefore has been required to avoid incorrect attribution of causation to COVID-19, with the apparent 'surge' perhaps simply caused by normal background viral infections, with higher reporting rates being encouraged by media attention. We feel that there is now sufficient evidence to be confident in a casual association.

We believe there is urgent need to add anosmia to the list of symptoms used in screening tools for possible COVID-19 infection. The current and further evolving evidence base makes it untenable to disregard this symptom any longer. France, Switzerland and the US have already adopted these measures, while other public health bodies are yet to acknowledge the link - the World Health Organization noted the early reports in March, but as yet have not updated their criteria. Use of loss of smell and taste as a marker of infection will be a very useful weapon in the COVID-19 fight, especially in countries with emerging pandemics where access to testing will be greatly limited.

\section{References}

1. Gane SB, Kelly C, Hopkins C. Isolated sudden onset anosmia in COVID-19 infection. A novel syndrome? Rhinology. 2020 Apr 2 doi: 10.4193/Rhin20.114. [Epub ahead of print].

2. Hopkins C, Surda P, Kumar N. Presentation of new onset anosmia during the COVID19 pandemic. Rhinology. 2020 Apr 11. doi: 10.4193/Rhin20.116. [Epub ahead of print].

3. Lechien JR, Chiesa-Estomba CM, De Siati DR, Horoi M, Le Bon SD, Rodriguez A, et al. Olfactory and gustatory dysfunctions as a clinical presentation of mild-to-moderate forms of the coronavirus disease (COVID19): a multicenter European study. Eur Arch Otorhinolaryngol. 2020 Apr 6. doi: 10.1007/ s00405-020-05965-1. [Epub ahead of print]

4. Kaye R CC, Kazahaya K, Brereton J et al. COVID-19 Anosmia Reporting tool: Initial Findings. Accepted preprint for Oto-HNS. 2020.

5. Stogbauer J, Wirkner K, Engel C, Moebus S, Pundt N, Teismann $\mathrm{H}$, et al. Prevalence and risk factors of smell dysfunction - a com- parison between five German populationbased studies. Rhinology. 2020;58(2):184-91.

6. Wang X, Zhang C, Xia X, Yang Y, Zhou C. Effect of gender on odor identification at different life stages: a meta-analysis. Rhinology. 2019;57(5):322-30.

7. https://www.medrxiv.org/content/10.1101/ 2020.04.15.20066472v1.

8. Salmon Ceron D HC, Bequignon E, Corre A, Canoui-Poitrine F, Papon JF. Anosmia without Nasal Obstruction: A Pathognomonic Sign of COVID-19 Infection. Press Release, March 28, 2020

9. Menni C VA, Freydin M, Ganesh S et al. Loss of smell and taste in combination with other symptoms is a strong predictor of COVID-19 infection. MedRxiv preprint server. 2020.

10. Yan CH, Faraji F, Prajapati DP, Boone CE, DeConde AS. Association of chemosensory dysfunction and Covid-19 in patients presenting with influenza-like symptoms. Int Forum Allergy Rhinol. 2020 Apr 12. doi: 10.1002/alr.22579. [Epub ahead of print]

11. Spinato G, Fabbris C, Polesel J, Cazzador
D, Borsetto D, Hopkins C, et al. Alterations in Smell or Taste in Mildly Symptomatic Outpatients With SARS-COV-2 Infection. Jama. 2020.

12. Moein ST, Hashemian SMR, Mansourafshar B, Khorram-Tousi A, Tabarsi P, Doty RL. Smell dysfunction: a biomarker for COVID-19. Int Forum Allergy Rhinol. 2020 Apr 17. doi: 10.1002/alr.22587. [Epub ahead of print]

13. Lotsch J, Hummel T. A data science-based analysis of seasonal patterns in outpatient presentations due to olfactory dysfunction. Rhinology. 2020;58(2):151-7.

Prof Claire Hopkins

ENT Dept Guy's Hospital

London

United kingdom

E-mail: clairehopkins@yahoo.com 